\title{
State of the Art Telemetric Implantable Sensors and Their Encapsulation
}

\author{
M. Kraft ${ }^{1}$, M. Görtz ${ }^{1}$, J. A. Müntjes ${ }^{2}$, W. Mokwa ${ }^{2}$, N. J. Cleven ${ }^{3}$, T. Schmitz-Rode ${ }^{3}$ \\ ${ }^{1}$ Fraunhofer Institut für Mikroelektronische Schaltungen und Systeme, Duisburg, Germany \\ michael.kraft@ims.fraunhofer.de \\ RWTH Aachen University, Institute of Materials in Electrical Engineering 1, Aachen, Germany \\ Department of Cardiovascular Engineering, Institute of Applied Medical Engineering, RWTH Aachen \\ University and University Hospital Aachen
}

\begin{abstract}
MEMS sensor have the potential to revolutionize medical implants. Their advantages are a small form factor, low power consumption and the ability to operate by telemetry. While some MEMS sensors, for example to measure pressure, have reached a high level of maturity, their encapsulation for long term implants remains problematic. Here, a novel encapsulation a technique is presented that combines flexibility with long term stability. It relies on a two layer approach: the outer thicker layer consists of a polymer, while the inner layer consists of a stack of thin layers which is long term stable and water vapor impenetrable. Furthermore, the discussed pressure sensor can be operated wirelessly, therefore no battery is required. The operation is described, and some initial measurement data on animal trials are presented.
\end{abstract}

Key words: pressure sensor, implant, wireless transmission, encapsulation

\section{Introduction}

Micro-electro-mechanical system (MEMS) sensors and devices offer tremendous potential for electrical implantable medical devices. Medical MEMS devices have been identified as a particular growth sector in the high-value MEMS market [1]. Well established are heart pace makers which can be made "intelligent" by the addition of sensors. Other examples include ventricular assist devices to support the heart function for patients with weakened heart conditions, retina implants to restore (at least partial) sight in blind people, cochlear implant to restore hearing, artificial bladder or bladder open/close systems, etc. All sensors for implants have in common that their form factor needs to be small, long term stability, biocompatibility and functionality needs to be guaranteed and their power consumption needs to be low so that battery life time is maximized, or wireless energy supply is possible. Furthermore, data communication needs to be wireless as skin penetrating cables are undesirable for obvious reasons.

MEMS pressure sensors can be used for many applications in implants, for example to continuously measure blood, brain or inner eye pressure. While suitable MEMS pressure sensing elements and their interface electronics are well-established technologies and have reached a mature state, their encapsulation for long term stability in implants remains problematic. A further challenge is the wireless energy supply and wireless data communication. For the work presented in the following a fully integrated pressure sensor fabricated in a surface micromachining process is used (see Fig. 1).

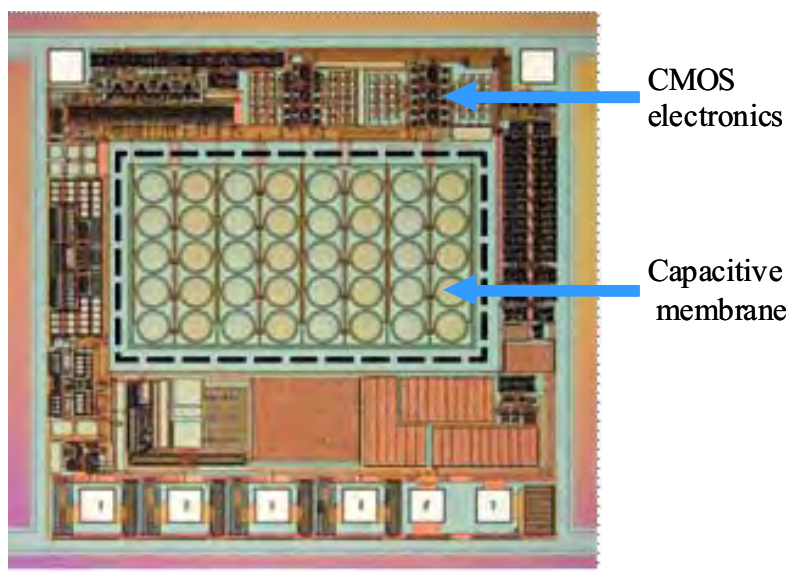

Fig. 1: Capacitive pressure sensor with integrated CMOS readout electronic. The capacitive membranes are arranged in a $5 \times 8$ array. 
In the following we report on recent developments on these two challenges and describe a flexible encapsulation technique that is fully bio-compatible and long term stable.

\section{Encapsulation}

Medically approved implants such as pace makers typically have a rigid casing made of steel, titanium or ceramics. However, for MEMS sensors this is often not advantageous as flexibility is a desired property to adjust to body anatomy and contours. Polymer based encapsulation (for example silicone) has been suggested for MEMS sensors, but this has long term stability problems as there is no polymer that is not fully water proof. Water penetration can lead to drift of the sensor signal or even complete failure of the MEMS sensor. We have developed an encapsulation technique for pressure sensors that combines flexibility with long term water resistance. The encapsulation consists of two main layers, as shown in fig. 2.

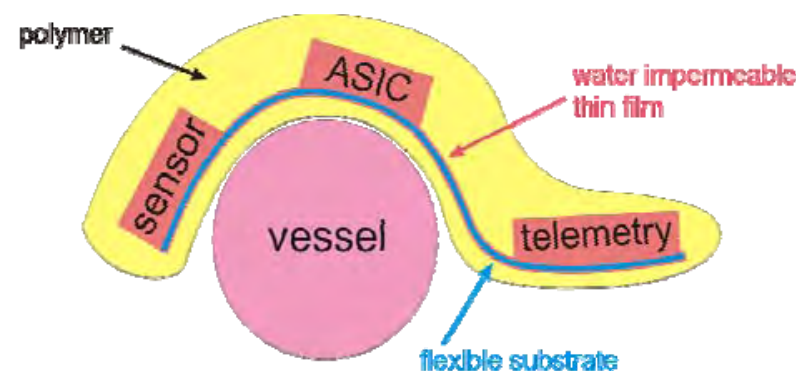

Fig. 2: Two layer encapsulation approach for flexible and small medical implants.

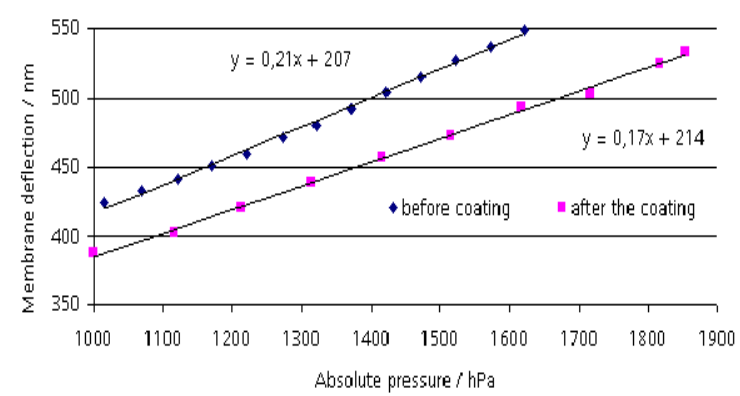

Fig. 3: Membrane deflection at its center as a function of the pressure before and after ONO and ALD deposition of 100nm $\mathrm{Ta}_{2} \mathrm{O}_{5}$.

Layer 1 is a hydrolytically stable polymer that is fully biocompatible and bio-stable (Med-6015 from Nusil). Underneath is a stack of several thin layers of a material that is water vapor impermeable. This two layer encapsulation technique allows combining flexibility and long term stability. The thin layer stack consisted of $750 \mathrm{~nm} \mathrm{SiO}$ with $2 \%$ Phosphor, $500 \mathrm{~nm} \mathrm{SiN}$,

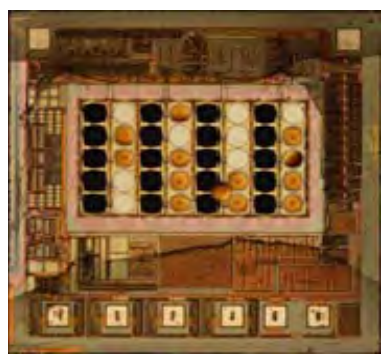

(a)

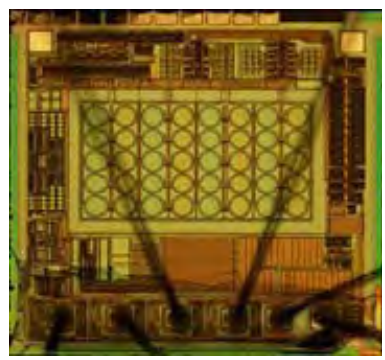

(b)
Fig. 4: Microphotograph of (a) a pressure sensor without additional protection layer after 135 hours and (b) with $A L D$ passivation layer and 442 hours storage in Phosphate buffered saline at a temperature of $120^{\circ} \mathrm{C}$.

and a further $100 \mathrm{~nm} \mathrm{SiO}_{2}$, all deposited by PECVD forming an ONO passivation layer system [2]. Following this, we used $\mathrm{Ta}_{2} \mathrm{O}_{5}$ acting as corrosion barrier and as water impermeable layer. $100 \mathrm{~nm} \quad \mathrm{Ta}_{2} \mathrm{O}_{5}$ was deposited by ALD (atomic layer deposition) at a process temperature of $250{ }^{\circ} \mathrm{C}$. As precursortantalum ethoxide and water were used. Thin ALD-layers are nearly free of pin holes and show excellent step coverage, which is a pre-requisite as a first encapsulation layer for MEMS sensors.

\section{Test Results}

The effect of the deposition of the thin layer on the membranes of the pressure sensor was investigated, as this effects the stiffness of the membrane and hence sensitivity. It was found that the deposition of the ONO passivation layer system and $100 \mathrm{~nm} \mathrm{Ta}_{2} \mathrm{O}_{5}$ had only little influence on membrane deflection. The membrane consisted of 1 um polysilicon. There is only a small shift in offset and an expected decrease in pressure sensitivity observable (see figure 3).

The encapsulation was tested for biocompatibility and stability, water impermeability and adhesion. Accelerated aging was studied by storing the device in an autoclave at elevated temperatures. Figure $4 a$ shows a photo of a pressure sensor chip without additional ALD passivation after storage in PBS (Phosphate buffered saline) in an autoclave for 135 hours at $120^{\circ} \mathrm{C}$. Strong corrosion is visible. In contrast, the sensor chip with additional ALD coating shows no corrosion even after 442 hours (figure $4 b)$. After the accelerated life time test, the chip was fully operational. Assuming an activation energy of $0.7 \mathrm{eV}$, a life time of more than 10 years at $37^{\circ} \mathrm{C}$ can be calculated. 


\section{Wireless Data Communication and Energy Supply}

Battery-free operation is a very desirable property for medical implants. One problem is that the pressure sensor itself has to be located deep inside the body, e.g. close to the heart. The electromagnetic field strength would be too high as potential health risk for the body tissue could not be excluded. Therefore, the transponder chip is connected to the sensor chip by wires and can thus be implanted under the skin, where it can be charged by electromagnetic fields of harmless intensity. Fig. 9 shows the pressure sensor and the transponder unit implanted in the artery fermoralis of a sheep. The transponder chip consists of a HF-front end, a state machine control unit, an EEPROM and a low power sensor readout with a 12 bit analogue to digital converter. Data transfer from the implant to the reader station is realized by amplitude modulation of the supplied energy carrier wave. The size of the transponder chip as shown in fig. 5 is $8 \mathrm{~mm}$ times $2.5 \mathrm{~mm}$.

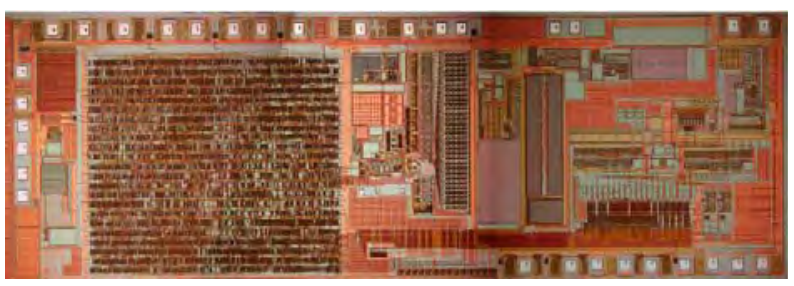

Fig. 5: Transponder chip receiving energy by inductive coupling from an external reader station. Chip size is $8 \mathrm{~mm}$ times $2.5 \mathrm{~mm}$.

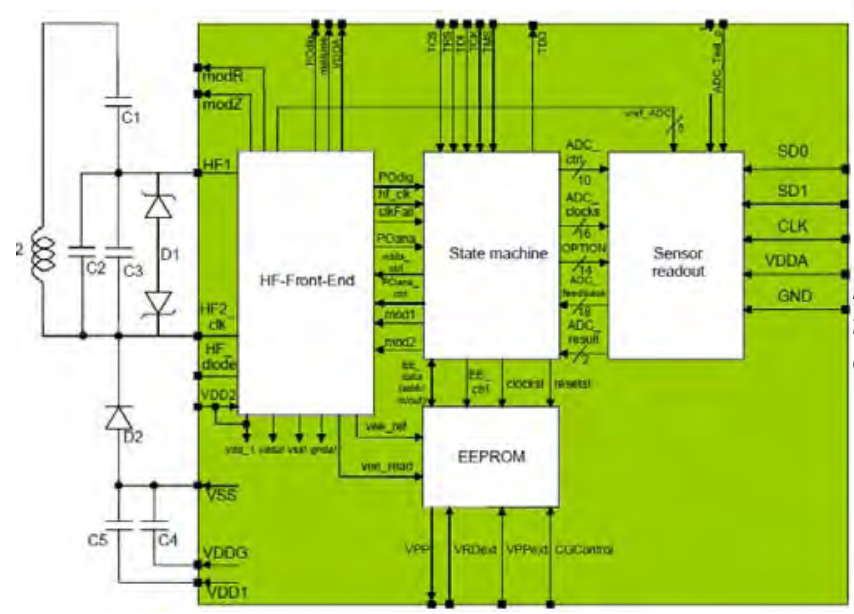

Fig. 6: Block diagram of the transpoder chip with required external components for telemetry.

In fig. 6, a block diagram of the transponder chip with the required external components for telemetric communication is shown.
The blocks for telemetry are located by the HFfront-end. The controlled supply voltage, required for sensor readout, is also generated by the HF-front-end. A state-machine (digital part of the chip) provides the protocol, which is necessary for data transmission of measured values. The internal EEPROM stores the ID and parts of the calibration coefficients. The setting data for offset and gain are transmitted by the bi-directional data-pads to the pressure sensor chip. After the measurement is finished the analogue output signals are transmitted, again by the data-pads, from the pressure sensor chip to the transponder chip, where they are digitalized by the sensor readout block. Next the digital data are sent by telemetry to the receiver coil of the external readout electronics.

\section{Test Results}

Before implantation, the pressure sensor has to be tested and calibrated. The calibration of the nonlinear and temperature dependent output of the pressure sensor chip is performed in a temperature controlled pressure chamber. A pressure characteristics and a typical calibration error, better than $1.4 \mathrm{hPa}$, are shown in fig.7 and fig. 8, respectively. The measurements curves are used to derive linearization coefficients that are stored in the on-chip EEPROM.

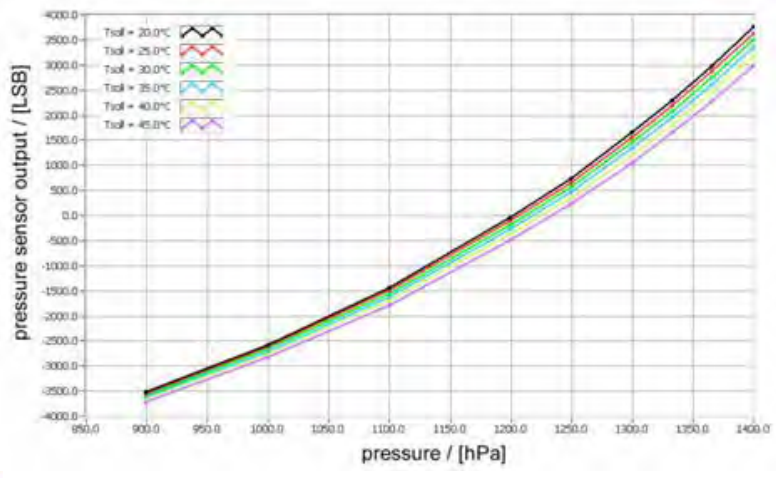

Fig. 7: Calibration test results for the pressure sensor tested at different temperature in a pressure chamber.

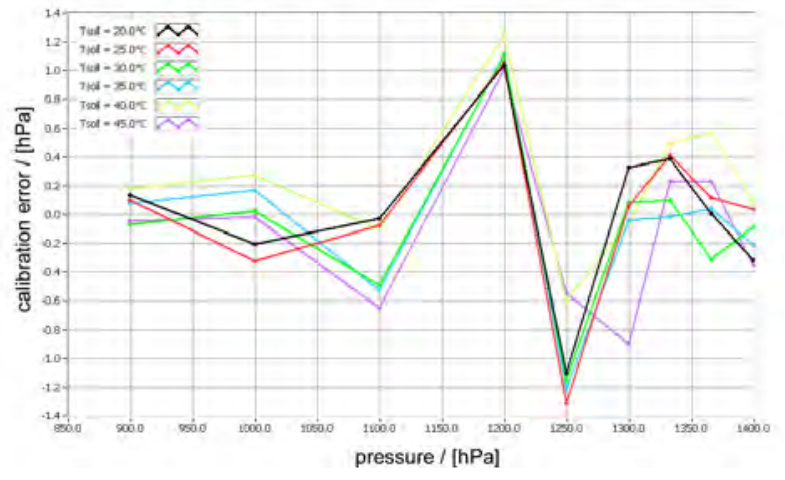

Fig. 8: Calibration error at different temperatures. 


\section{In vivo measurements}

In vivo experiments with implants have been successfully completed. The implant was placed in the artery femoralis of a sheep. The position of the implant in the sheep is shown in fig. 9 on the left side as an angiography and on the right side as a x-ray photograph.

The wirelessly recorded data of the implant (blue signal in fig. 10) match the data of a reference sensor (red signal in fig. 10).

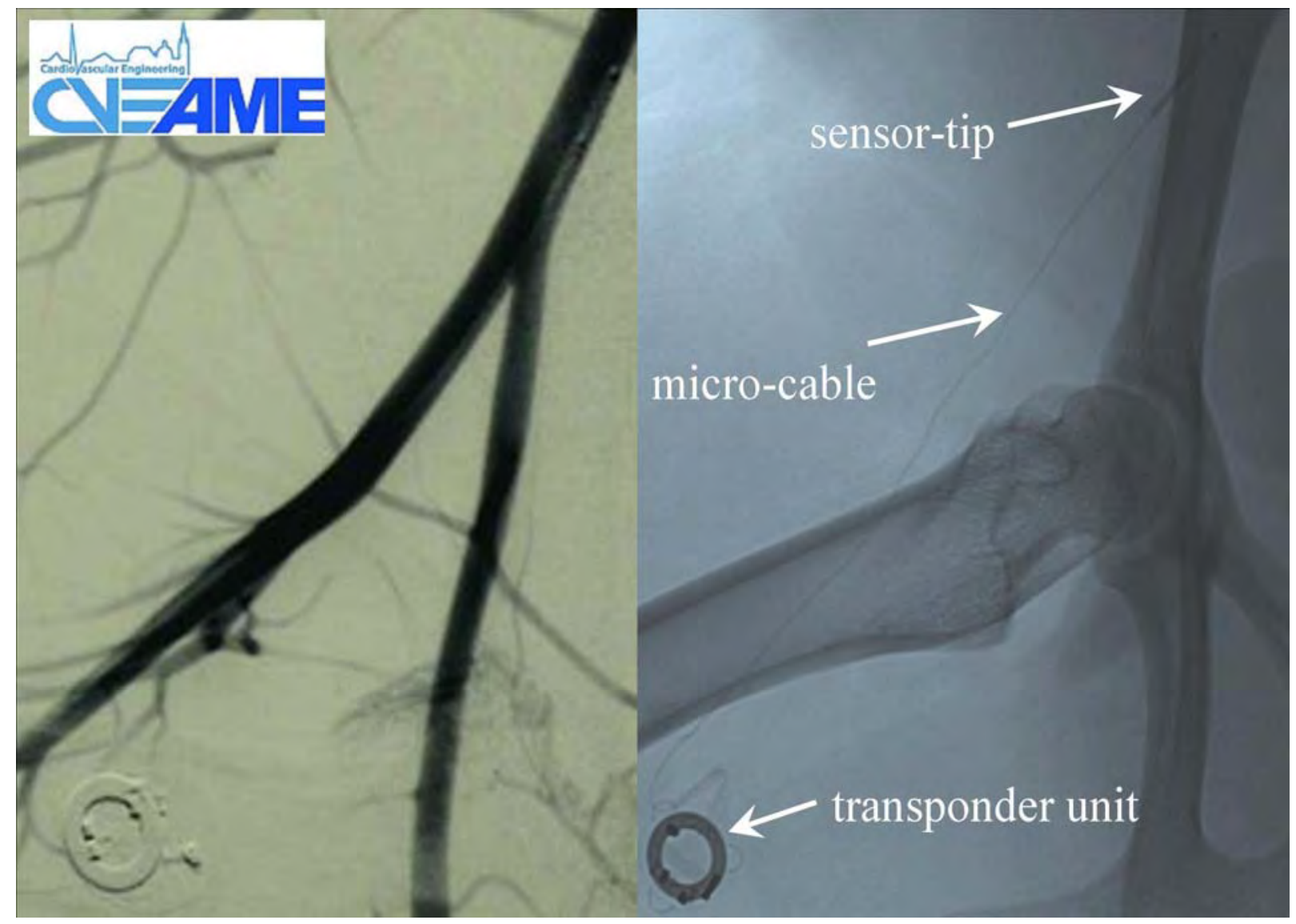

Fig. 9 Position of the implant (left: angiography- right: x-ray-photograph)

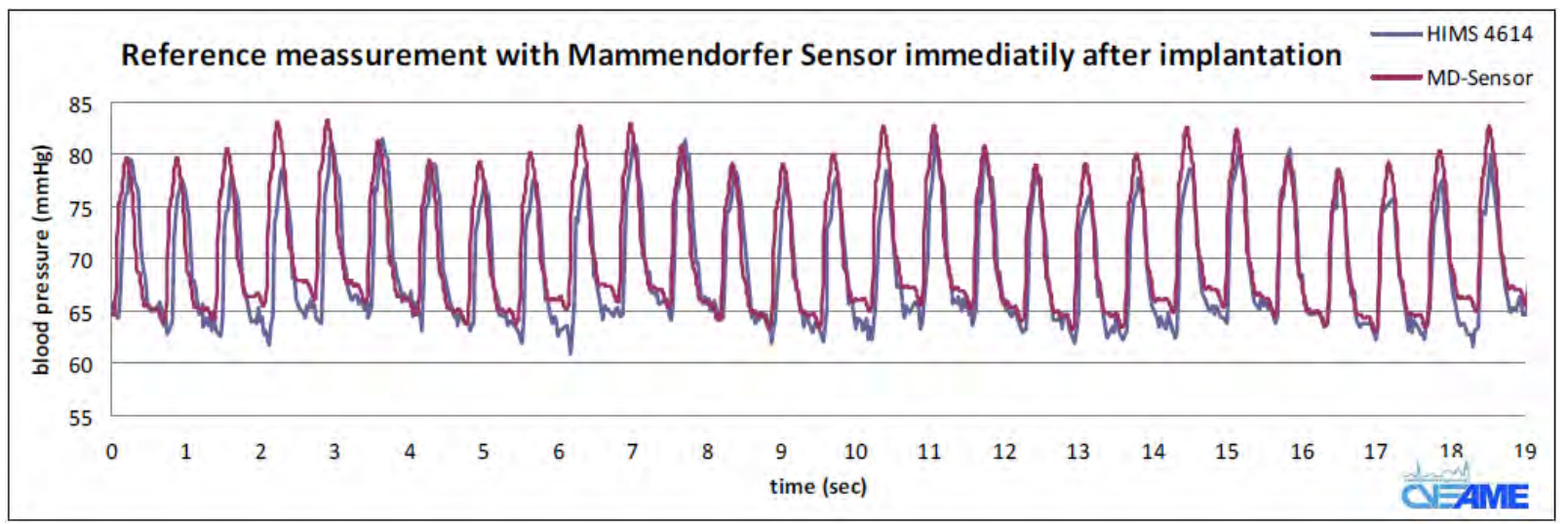

Fig. 10: In-vivo blood pressure measuring results. The wireless MEMS data is shown in the blue curve, whereas the red signal shows data from a reference sensor.

\section{Conclusions}

The paper reports on state-of-the-art pressure sensors for medical devices. A novel encapsulation technique has been developed, relying on two main layers: silicone and a thin layer stack consisting of $\mathrm{SiN}, \mathrm{SiO}_{2}$ and $\mathrm{Ta}_{2} \mathrm{O}_{5}$.
Accelerated aging tests showed excellent long term stability.

The pressure sensor is connected to a separate transponder chip located close to the skin. Data transmission and power is supplied by inductive coupling. The implant was tested to measure 
blood pressure in a sheep. Initial test results agree very well with data from a reference sensor.

\section{References}

[1] http://www.giiresearch.com/report/bc261431mems-devices-global-medical-markets.html. Accessed 22.2.13.

[2] M. Hofmann, S. Kambor, C. Schmidt, D. Grambole, J. Rentsch, S. W. Glunz, and R. Preu, PECVD-ONO: a new deposited firing stable rear surface passivation layer system for crystalline silicon solar cells. Advances in Opto Electronics, Vol. 2008 (2008), doi: 10.1155/2008/485467 